\title{
Extended Kalman Filter for Vehicle Tracking using Road Surface Vibration Measurements
}

\author{
Roland Hostettler, Wolfgang Birk, and Magnus Lundberg Nordenvaad
}

This is a post-print of a paper published in 51st IEEE Conference on Decision and Control. When citing this work, you must always cite the original article:

R. Hostettler, W. Birk, and M. Lundberg Nordenvaad, "Extended Kalman filter for vehicle tracking using road surface vibration measurements," in 51st IEEE Conference on Decision and Control, Maui, December 2012, accepted for publication

DOI:

10.1109/CDC.2012.6426451

\section{Copyright:}

(c) 2015 IEEE. Personal use of this material is permitted. Permission from IEEE must be obtained for all other uses, in any current or future media, including reprinting/republishing this material for advertising or promotional purposes, creating new collective works, for resale or redistribution to servers or lists, or reuse of any copyrighted component of this work in other works. 


\title{
Extended Kalman Filter for Vehicle Tracking using Road Surface Vibration Measurements
}

\author{
Roland Hostettler, Wolfgang Birk, and Magnus Lundberg Nordenvaad
}

\begin{abstract}
This paper addresses a novel method for vehicle tracking using an extended Kalman filter and measurements of road surface vibrations from a single accelerometer. First, a measurement model for vibrations caused by vehicular road traffic is developed. Then the identifiability of the involved parameters is analyzed. Finally, the measurement model is combined with a constant speed motion model and the Kalman filter is derived. Simulation and measurement results indicate that the approach is feasible and show where further development is needed.
\end{abstract}

\section{INTRODUCTION}

Vehicle tracking and speed estimation is an important issue in traffic control and intelligent transportation systems (ITS) applications. Technologies like radar and vision systems are very popular for this purpose. Such solutions have the advantage of providing accurate and diverse information such as speed, heading direction, and more. Drawbacks include the generally rather large installation size and the computation power needed [2], [3], [4].

On the other hand, recent advances in microelectronics have produced a range of low-cost sensors that complement the existing technologies. For example, microphones, magnetometers, or accelerometers are increasingly popular for new solutions. These types of sensors are not only low-cost but also small and can be integrated easily. Different approaches for vehicle tracking and speed estimation using a combination of these sensors have been proposed [5], [6], [7]. Most of the proposed methods make use of two sensors which requires good synchronization, communication between the sensors, and reliable vehicle re-identification.

The aim of this paper is to explore the potential of using a single accelerometer for vehicle tracking and speed estimation in order to address the above mentioned drawbacks. Based on previous research, a signal model is developed which is then used in an extended Kalman filter (EKF) for vehicle tracking. As a by-product of the tracking, a speed estimate is automatically obtained.

The remainder of this paper is organized as follows. Section II introduces the signal model as well as the preprocessing required in order to recover the signal. Based on this, the EKF is presented in Section III and evaluated in

Financial support from GEVEKO AB and the Swedish Governmental Agency for Innovation Systems (VINNOVA) are gratefully acknowledged.

Roland Hostettler and Wolfgang Birk are with the Department of Computer Science, Electrical and Space Engineering, Luleå University of Technology, Luleå, Sweden, \{rolhos, wolfgang\}@ltu. se.

Magnus Lundberg Nordenvaad is with the Department of Information Technology, Uppsala University, Uppsala, Sweden, magnus. lundberg-nordenvaaddit.uu.se. simulations and on real measurement data in Section IV. Some concluding remarks are given in Section V.

\section{SIGNAL MODEL}

\section{A. Wave Propagation}

The pavement as a wave propagation medium has been studied before [8], [9]. A simplified, semi-parametric model for the pavement's frequency response function $H_{0}(\boldsymbol{r}, i \omega)$ is given by

$$
H_{0}(\boldsymbol{r}, i \omega)=\frac{\alpha(\omega)}{\sqrt{|\boldsymbol{r}|}} e^{-\beta(\omega)|\boldsymbol{r}|} e^{-i\left(\frac{\omega|\boldsymbol{r}|}{c_{p}(\omega)}+\varphi_{0}\right)}
$$

where $\boldsymbol{r}=\left[\begin{array}{ll}r_{x} & r_{y}\end{array}\right]^{T}$ is the distance vector between the source and the sensor, $\omega$ is the angular frequency, $c_{p}$ is the wave propagation speed in the material, $\varphi_{0}$ is a constant phase shift, and the constants $\alpha$ and $\beta$ are gain and damping coefficients, respectively. The model accounts for a phase shift caused by the propagation delay $|\boldsymbol{r}| / c_{p}, 1 / \sqrt{|\boldsymbol{r}|}$ damping where $\alpha$ is the corresponding gain, and exponential decay in $|\boldsymbol{r}|$ with the decay constant $\beta$ [10].

\section{B. Excitation}

An external force acting on the pavement excites different Lamb wave modes as it was shown in [10]. Assuming that the vehicle-road interaction of a target moving along the road (Figure 1) can be seen as a broadband excitation, a set of these different modes will be excited and measured on the road surface. Picking one specific mode centered around its principal frequency $\omega_{0}$ (e.g. the $A_{0}$-mode which is mainly associated with flexural plate bending, see [11]), the moving excitation can be modeled as

$$
f(t)=N_{0} \delta\left(r_{x}-v t\right) \delta\left(r_{y}-r_{y, 0}\right) \cos \left(\omega_{0} t\right)
$$

where $N_{0}$ is the excitation magnitude, $\delta(t)$ is the Dirac delta function, $r_{x}$ and $r_{y}$ are the longitudinal and lateral displacements according to Figure 1, $v$ is the vehicle's speed, and $r_{y, 0}$ is the lateral distance between the source trajectory and the sensor. $N_{0}$ is a combination of the static ground force (vehicle mass) and the dynamic force created by the tire-road interaction which depends on the vehicle speed, the road profile, and the filtering characteristics of the vehicle suspension [12]. Note that the excitation is not a deterministic sinusoidal source by itself but is modeled this way due to the wave propagation properties of the pavement.

In order to account for the vehicle motion in the whole $\mathrm{X}$-Y-plane, the sum of the responses of all the excited points 


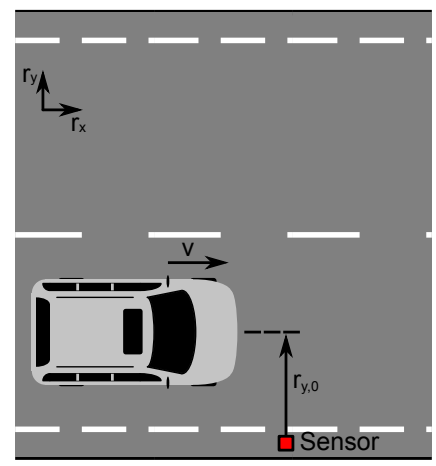

Fig. 1. Illustration of the coordinate system relative to the sensor: The origin is at the sensor's location, the y-axis in the lateral and the x-axis in the longitudinal directions, respectively.

has to be taken, that is,

$$
z(t)=\iint_{-\infty}^{\infty} f(t) * h_{0}(\boldsymbol{r}, t) d r_{x} d r_{y}
$$

where $*$ denotes convolution and $h_{0}(\boldsymbol{r}, t)$ is the impulse response of (1). Using (1)-(3) yields

$$
z(t)=N_{0} \frac{\alpha}{\sqrt{|\boldsymbol{r}|}} e^{-\beta|\boldsymbol{r}|} \cos \left(\omega_{0} t-\frac{\omega_{0}|\boldsymbol{r}|}{c_{p}}-\varphi_{0}\right)
$$

with $\boldsymbol{r}=\left[\begin{array}{ll}v t & r_{y, 0}\end{array}\right]^{T}, \alpha=\alpha\left(\omega_{0}\right), \beta=\beta\left(\omega_{0}\right)$, and $c_{p}=$ $c_{p}\left(\omega_{0}\right)$.

Since the phase velocity $c_{p}$ is rather large, the propagation delay $|\boldsymbol{r}| / c_{p}$ can be neglected and (4) becomes approximately

$$
z(t) \approx N_{0} \frac{\alpha}{\sqrt{|\boldsymbol{r}|}} e^{-\beta|\boldsymbol{r}|} \cos \left(\omega_{0} t-\varphi_{0}\right)
$$

Each wheel of a vehicle is an individual source and thus, one vehicle actually excites the road at $2 P$ points where $P$ is the number of axles. Assuming that one axle's wheels can be lumped together, the measured signal becomes

$$
z_{p}(t) \approx \sum_{p=1}^{P} N_{0} \frac{\alpha}{\sqrt{\left|\boldsymbol{r}_{p}\right|}} e^{-\beta\left|\boldsymbol{r}_{p}\right|} \cos \left(\omega_{0} t-\varphi_{0}\right) .
$$

$\boldsymbol{r}_{p}$ can be rewritten as

$$
\boldsymbol{r}_{p}=\boldsymbol{r}_{p-1}-\left[\begin{array}{c}
l_{p} \\
0
\end{array}\right] \text { for } p=2, \ldots, P
$$

where $l_{p}$ is the wheel base between the $(p-1)$-th and the $p$-th axle. Considering the most common group of vehicles, two-axled passenger cars and small trucks yields

$z_{2}(t) \approx \kappa\left(\frac{1}{\sqrt{\left|\boldsymbol{r}_{1}\right|}} e^{-\beta\left|\boldsymbol{r}_{1}\right|}+\frac{1}{\sqrt{\left|\boldsymbol{r}_{2}\right|}} e^{-\beta\left|\boldsymbol{r}_{2}\right|}\right) \cos \left(\omega_{0} t-\varphi_{0}\right)$

with $\boldsymbol{r}_{1}=\left[\begin{array}{ll}v t & r_{y, 0}\end{array}\right]^{T}, \boldsymbol{r}_{2}=\boldsymbol{r}_{1}-\left[\begin{array}{ll}l & 0\end{array}\right]^{T}$, and $\kappa=N_{0} \alpha$.

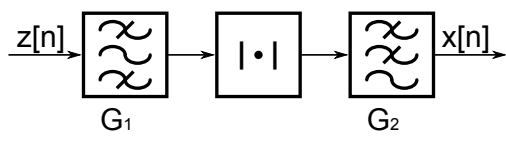

Fig. 2. Preprocessing used to recover the envelope from the measured Lamb wave mode. After filtering out the desired mode, it is down-mixed and low-pass filtered to obtain the envelope.

\section{Preprocessing}

Assuming that a noisy, sampled version of (7) is measured using an accelerometer mounted on the road surface, the measured signal is

$$
y[n]=z_{2}\left(n T_{s}\right)+e\left(n T_{s}\right)=z_{2}[n]+e[n]
$$

where $T_{s}$ is the sampling time and $e[n] \sim \mathcal{N}\left(0, \sigma_{e}^{2}\right)$. (7) can be thought of as a sum of two amplitude modulated signals and its envelope can be recovered using a simple envelope detector as illustrated in Figure 2. First, the desired mode around $\omega_{0}$ is isolated using the bandpass filter $G_{1}$. By taking the absolute value, that mode is down-mixed to be centered around $\omega=0$ and finally, the higher order components (around $2 \omega_{0}, 3 \omega_{0}$, etc.) are removed by the lowpass filter $G_{2}$. The recovered pulse is then described by

$$
x[n]=\underbrace{\kappa\left(\frac{1}{\sqrt{\left|\boldsymbol{r}_{1}\right|}} e^{-\beta\left|\boldsymbol{r}_{1}\right|}+\frac{1}{\sqrt{\left|\boldsymbol{r}_{2}\right|}} e^{-\beta\left|\boldsymbol{r}_{2}\right|}\right)}_{h[n ; \boldsymbol{\theta}]}+w[n]
$$

where $w[n]$ is the disturbance term which is no longer zero mean Gaussian noise due to the nonlinearity. This method is similar to the one used in [13]. However, the square-nonlinearity used there was replaced by an absolutevalue nonlinearity in order to avoid cross terms between the individual pulses and the pulses and noise.

\section{Identifiability}

Assuming that none of the parameters in $\boldsymbol{\theta}=$ $\left[\begin{array}{llllll}r_{x} & r_{y} & l & v & \kappa & \beta\end{array}\right]^{T}$ is known, one can analyze the identifiability of the individual parameters [14].

The elements of the Fisher information matrix $\boldsymbol{I}(\boldsymbol{\theta})$ under the assumption of additive white Gaussian noise are given by

$$
[\boldsymbol{I}(\boldsymbol{\theta})]_{i j}=\frac{1}{\sigma_{w}^{2}} \sum_{n=0}^{N-1} \frac{\partial h[n ; \boldsymbol{\theta}]}{\partial \theta_{i}} \frac{\partial h[n ; \boldsymbol{\theta}]}{\partial \theta_{j}} .
$$

In general, this yields a full rank matrix. However, the condition number $\gamma$ becomes very large due to $\kappa$ and $\beta$. By removing these parameters and letting $\tilde{\boldsymbol{\theta}}=\left[\begin{array}{llll}r_{x} & r_{y} & l & v\end{array}\right]^{T}$ the information matrix becomes much better conditioned and thus the estimation problem becomes easier to solve. This is illustrated in Figure 3 where the condition number of $\boldsymbol{I}(\boldsymbol{\theta})$ and $\boldsymbol{I}(\tilde{\boldsymbol{\theta}})$ as functions of $\kappa$ and $\beta$ for a vehicle passing with $v=10 \mathrm{~m} / \mathrm{s}, r_{y, 0}=1 \mathrm{~m}$, and $l=2.5 \mathrm{~m}$ are shown.

Note that $\boldsymbol{I}(\boldsymbol{\theta})$ can be expressed as

$$
\boldsymbol{I}(\boldsymbol{\theta})=\frac{1}{\sigma_{w}^{2}}\left[\begin{array}{cc}
\boldsymbol{I}(\tilde{\boldsymbol{\theta}}) & \boldsymbol{I}_{\tilde{\boldsymbol{\theta}} \overline{\boldsymbol{\theta}}} \\
\boldsymbol{I}_{\tilde{\boldsymbol{\theta}} \overline{\boldsymbol{\theta}}}^{T} & \boldsymbol{I}(\overline{\boldsymbol{\theta}})
\end{array}\right]
$$



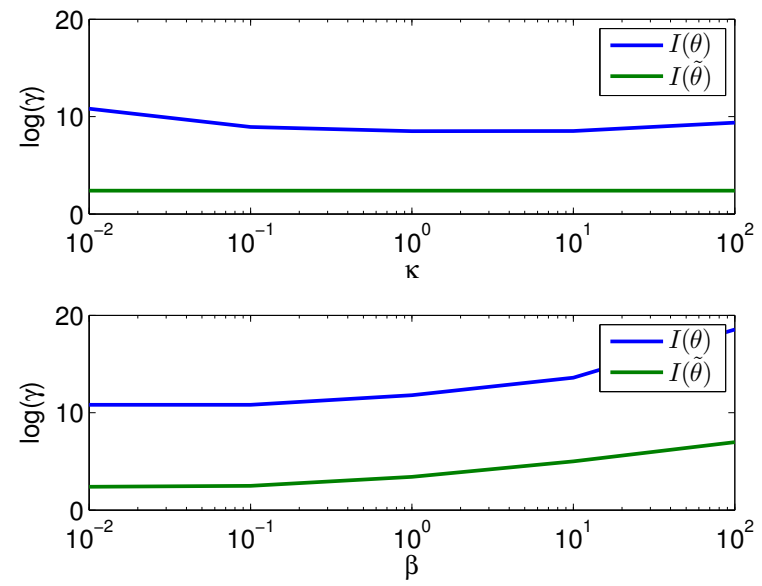

Fig. 3. Logarithm of the condition number for the full estimation problem with $\boldsymbol{\theta}=\left[\begin{array}{llllll}r_{x} & r_{y} & l & v & \kappa & \beta\end{array}\right]^{T}$ versus the simplified problem with $\tilde{\boldsymbol{\theta}}=\left[\begin{array}{llll}r_{x} & r_{y} & l & v\end{array}\right]^{T}$ for varying $\kappa$ (top) and $\beta$ (bottom).

with $\overline{\boldsymbol{\theta}}=\left[\begin{array}{ll}\kappa & \beta\end{array}\right]^{T}$. Then, the elements of $\boldsymbol{I}_{\tilde{\boldsymbol{\theta}} \overline{\boldsymbol{\theta}}}$ give an indication about the mutual information of the parameters in $\tilde{\boldsymbol{\theta}}$ and $\overline{\boldsymbol{\theta}}$. For example, for the case outlined above with $\kappa=0.01$ and $\beta=0.1$, the elements of (11) are

$$
\begin{aligned}
& \boldsymbol{I}(\tilde{\boldsymbol{\theta}})=10^{-3} \cdot\left[\begin{array}{cccc}
0.45 & 0 & -0.23 & 0.90 \\
0 & 1.35 & 0.15 & 0.11 \\
-0.23 & 0.15 & 0.21 & -0.48 \\
0.90 & 0.11 & -0.48 & 1.87
\end{array}\right] \\
& \boldsymbol{I}(\overline{\boldsymbol{\theta}})=\left[\begin{array}{cc}
130.6 & -3.8 \\
-3.8 & 0.2
\end{array}\right] \\
& \boldsymbol{I}_{\overline{\boldsymbol{\theta}} \tilde{\boldsymbol{\theta}}}=\left[\begin{array}{cccc}
0 & -0.38 & -0.03 & -0.06 \\
0 & 0.01 & 0 & 0
\end{array}\right]^{T}
\end{aligned}
$$

which shows that there exists a rather strong connection between $\kappa$ and $r_{y}, l$, and $v$. Furthermore, also $\kappa$ and $\beta$ contain mutual information.

An intuitive explanation for this is that the scaling parameters $\kappa$ and $\beta$ can be included in $|\boldsymbol{r}|$. This problem originates from the fact that it is generally difficult to distinguish between a light vehicle close to the sensor (small $N_{0}$ and $\left.r_{y, 0}\right)$ and a heavy vehicle farther away (large $N_{0}$ and $r_{y, 0}$ ). Usually, this problem is treated by adding a second sensor, preferably on the opposite side of the road, with which's information this duality can be resolved.

If no second sensor is available as in the proposed solution, a different strategy for handling these parameters has to be found. Recalling that $\kappa=N_{0} \alpha$ and identifying that $\alpha$ and $\beta$ are material properties, it becomes apparent that these parameters could easily be determined by using a few calibration measurements. Furthermore, $N_{0}$ (or even $\kappa$ ) could be eliminated by using a normalization such as a p-norm $\|z\|_{p}$. However, due to the form of (9), no closed form solution for the corresponding theoretical $\|z\|_{p}$ can be found.

Nevertheless, for demonstrating the feasibility of the proposed method, it is thus assumed that both $\kappa$ and $\beta$ can be regarded as tuning parameters that can be obtained through calibration and the issue of dealing with $N_{0}$ is left as an open research question.

\section{EXTENDED KALMAN FILTER}

\section{A. Dynamic process model}

The dynamic process model consists of a constant speed motion model with the driving noise in the acceleration term. The dynamic equations are given by

$$
\begin{aligned}
& r_{x}[n]=r_{x}[n-1]+T_{s} v[n-1]+\frac{T_{s}^{2}}{2} u[n] \\
& r_{y}[n]=r_{y}[n-1] \\
& l[n]=l[n-1] \\
& v[n]=v[n-1]+T_{s} u[n]
\end{aligned}
$$

From (13) we find the state vector as $s[n]=$ $\left[\begin{array}{llll}r_{x}[n] & r_{y}[n] & l[n] & v[n]\end{array}\right]^{T}$. The driving noise is assumed to be of the form $u[n] \sim \mathcal{N}\left(0, \sigma_{u}^{2}\right)$. Finally, (13) can be rewritten on state-space form as

$$
\boldsymbol{s}[n]=\underbrace{\left[\begin{array}{cccc}
1 & 0 & 0 & T_{s} \\
0 & 1 & 0 & 0 \\
0 & 0 & 1 & 0 \\
0 & 0 & 0 & 1
\end{array}\right]}_{\boldsymbol{A}} \boldsymbol{s}[n-1]+\underbrace{\left[\begin{array}{c}
\frac{T_{s}^{2}}{2} \\
0 \\
0 \\
T_{s}
\end{array}\right]}_{\boldsymbol{B}} u[n]
$$

\section{B. Sensing matrix}

As shown in Section II, the measured signal is given by (9) and thus the measurement function $h(s[n])$ is a nonlinear function of the states $\boldsymbol{s}[n]$ given by

$$
h(\boldsymbol{s}[n])=\kappa\left(\frac{1}{\sqrt{\left|\boldsymbol{r}_{1}\right|}} e^{-\beta\left|\boldsymbol{r}_{1}\right|}+\frac{1}{\sqrt{\left|\boldsymbol{r}_{2}\right|}} e^{-\beta\left|\boldsymbol{r}_{2}\right|}\right)
$$

Thus, an extended Kalman filter is used and the sensing matrix $\boldsymbol{H}$ becomes the Jacobian of (15) evaluated at the predicted state, that is, by letting $\boldsymbol{s}[n]=\hat{\boldsymbol{s}}[n \mid n-1]$. The elements of $\boldsymbol{H}[n](\boldsymbol{s}[n])$ are

$$
\begin{aligned}
& {[\boldsymbol{H}[n](\boldsymbol{s}[n])]_{11}=\frac{\partial h(\boldsymbol{s}[n])}{\partial r_{x}}} \\
& =-\kappa\left(\frac{1}{\sqrt{\left|\boldsymbol{r}_{1}\right|}} e^{-\beta\left|\boldsymbol{r}_{1}\right|} \frac{r_{x}}{\left|\boldsymbol{r}_{1}\right|}\left(\frac{1}{2\left|\boldsymbol{r}_{1}\right|}+\beta\right)\right. \\
& \left.\quad+\frac{1}{\sqrt{\left|\boldsymbol{r}_{2}\right|}} e^{-\beta\left|\boldsymbol{r}_{2}\right|} \frac{r_{x}-l}{\left|\boldsymbol{r}_{2}\right|}\left(\frac{1}{2\left|\boldsymbol{r}_{2}\right|}+\beta\right)\right) \\
& {[\boldsymbol{H}[n](\boldsymbol{s}[n])]_{12}=\frac{\partial h(\boldsymbol{s}[n])}{\partial r_{y}}} \\
& =-\kappa r_{y}\left(\frac{1}{\sqrt{\left|\boldsymbol{r}_{1}\right|}} e^{-\beta\left|\boldsymbol{r}_{1}\right|} \frac{1}{\left|\boldsymbol{r}_{1}\right|}\left(\frac{1}{2\left|\boldsymbol{r}_{1}\right|}+\beta\right)\right. \\
& \left.\quad+\frac{1}{\sqrt{\left|\boldsymbol{r}_{2}\right|}} e^{-\beta\left|\boldsymbol{r}_{2}\right|} \frac{1}{\left|\boldsymbol{r}_{2}\right|}\left(\frac{1}{2\left|\boldsymbol{r}_{2}\right|}+\beta\right)\right)
\end{aligned}
$$


TABLE I

EXTENDED KALMAN FILTER

Initialization: $\hat{\boldsymbol{s}}[-1 \mid-1]=\mathrm{E}\{\boldsymbol{s}\}, \boldsymbol{M}[-1 \mid-1]=\boldsymbol{C}_{\boldsymbol{s}}$

1. Prediction update:

$\hat{\boldsymbol{s}}[n \mid n-1]=A \hat{\boldsymbol{s}}[n-1 \mid n-1]$

$\boldsymbol{M}[n \mid n-1]=\boldsymbol{A} \boldsymbol{M}[n-1 \mid n-1] \boldsymbol{A}^{T}+\boldsymbol{B} \boldsymbol{Q} \boldsymbol{B}^{T}$

2. Calculate gain:

$\boldsymbol{K}[n]=\boldsymbol{M}[n \mid n-1] \boldsymbol{H}^{T}[n]\left(\boldsymbol{C}+\boldsymbol{H}[n] \boldsymbol{M}[n \mid n-1] \boldsymbol{H}^{T}[n]\right)^{-1}$

3. Estimation update:

$\hat{\boldsymbol{s}}[n \mid n]=\hat{\boldsymbol{s}}[n \mid n-1]+\boldsymbol{K}[n](x[n]-h(\hat{\boldsymbol{s}}[n \mid n-1])$

$\boldsymbol{M}[n \mid n]=(\boldsymbol{I}-\boldsymbol{K}[n] \boldsymbol{H}[n]) \boldsymbol{M}[n \mid n-1]$

$$
\begin{aligned}
& {[\boldsymbol{H}[n](\boldsymbol{s}[n])]_{13}=\frac{\partial h(\boldsymbol{s}[n])}{\partial l}} \\
& \quad=\kappa \frac{1}{\sqrt{\left|\boldsymbol{r}_{2}\right|}} e^{-\beta\left|\boldsymbol{r}_{2}\right|} \frac{\boldsymbol{r}_{x}-l}{\left|\boldsymbol{r}_{2}\right|}\left(\frac{1}{2\left|\boldsymbol{r}_{2}\right|}+\beta\right) \\
& {[\boldsymbol{H}[n](\boldsymbol{s}[n])]_{14}=\frac{\partial h(\boldsymbol{s}[n])}{\partial v}=0}
\end{aligned}
$$

\section{Extended Kalman Filter}

The EKF is summarized in Table I [15] and can now be used to track the vehicle and estimate its parameters. The individual estimates $\hat{\boldsymbol{s}}[n \mid n]$ as well as the corresponding covariance matrix $M[n \mid n]$ are stored during the estimation. Finally, the best estimates are chosen where the Frobenius norm of $\boldsymbol{M}[n \mid n]$ is minimal after the complete vehicle passage.

\section{State initialization}

In order for the Kalman filter to yield good results, good state initialization is necessary. Reasonable choices for the a priori distributions of $\hat{\boldsymbol{s}}[-1 \mid-1]$, i.e. the initial values are:

- $r_{x} \sim \mathcal{N}\left(-15 \mathrm{~m}, 5 \mathrm{~m}^{2}\right)$ - The typical sensing range of an accelerometer and a rather large uncertainty.

- $r_{y} \sim \mathcal{N}\left(1 \mathrm{~m}, 0.5 \mathrm{~m}^{2}\right)$ - A reasonable distance from the road edge to the car and a moderate distribution across the nearby driving lane.

- $l \sim \mathcal{N}\left(2.5 \mathrm{~m}, 0.5 \mathrm{~m}^{2}\right)$ - The typical wheelbase of a passenger car and a moderate variation.

- $v \sim \mathcal{N}\left(0 \mathrm{~m} / \mathrm{s}, 10(\mathrm{~m} / \mathrm{s})^{2}\right)$ - Zero initial speed to avoid aggregation of integral errors and a rather large variation in order to enable evolution.

\section{RESULTS AND DISCUSSION}

\section{A. Simulation}

In order to verify the validity of the proposed Kalman filter, it is evaluated in simulations with known parameters first. The initial values were chosen as described in Section III-D above, that is,

$$
\mathrm{E}\{\boldsymbol{s}\}=\left[\begin{array}{c}
-15 \\
1 \\
2.5 \\
0
\end{array}\right] \text { and } \boldsymbol{C}_{\boldsymbol{s}}=\left[\begin{array}{cccc}
5 & 0 & 0 & 0 \\
0 & 0.5 & 0 & 0 \\
0 & 0 & 0.5 & 0 \\
0 & 0 & 0 & 10
\end{array}\right]
$$

and $\kappa=0.1$ and $\beta=0.5$. White Gaussian measurement noise with a variance of $\sigma_{w}^{2}=10^{-5}$ was added.

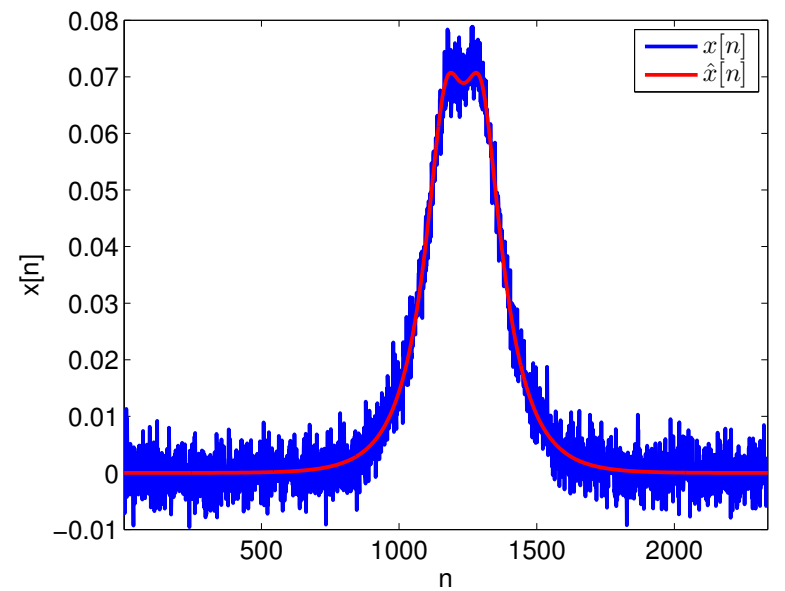

Fig. 4. Simulated and estimated signal.

The simulation results are shown in Figure 4 and Figure 5. Figure 4 shows the simulated signal together with the reconstructed signal $\hat{x}[n]$. The resulting parameters are $\hat{r}_{y}=1.2 \mathrm{~m}$ (true value $\left.r_{y}=1.2 \mathrm{~m}\right), \hat{l}=2.3 \mathrm{~m}(l=2.2 \mathrm{~m})$, and $\hat{v}=17.5 \mathrm{~m} / \mathrm{s}(v=17.1 \mathrm{~m} / \mathrm{s})$.

Figure 5 depicts how the individual parameters develop and converge towards the true trajectories. Furthermore, the $2 \sigma$ confidence intervals are shown for the corresponding diagonal elements of the covariance matrix $\boldsymbol{M}[n \mid n]$. As expected, the confidence interval narrows as soon as $\hat{\boldsymbol{s}}[n \mid n]$ converges.

Using the same simulation setup as described before, a total of 1,000 simulations were run in order to evaluate the convergence of the filter. Figure 6 shows the relative frequency of the estimated parameters relative to the true value, i.e. $\hat{\theta}_{i} / \theta_{i}$ for the three parameters $r_{y}, l$, and $v$ where the two outermost bins include the intevals $(-\infty, 0.5]$ and $[1.5, \infty)$, respectively. All the three parameters are centered around the true value. The expected values and the corresponding variances are

$$
\begin{aligned}
& \mathrm{E}\left\{\hat{r}_{y} / r_{y}\right\}=0.96, \operatorname{var}\left\{\hat{r}_{y} / r_{y}\right\}=0.042 \\
& \mathrm{E}\{\hat{l} / l\}=1.11, \operatorname{var}\{\hat{l} / l\}=3.7 \\
& \mathrm{E}\{\hat{v} / v\}=0.98, \operatorname{var}\{\hat{v} / v\}=0.008
\end{aligned}
$$

The comparably larger variance of $\hat{l} / l$ is explained by a few outliers (for example one at $-55.15 \mathrm{~m}$ ). Also, the expected values show that the estimates are slightly biased which can also be observed in the asymptotic behaviour in Figure 5.

\section{B. Measurements}

In order to practically test the feasibility, road vibrations were measured using a tri-axial accelerometer. The accelerometer was placed on the road side and traffic was measured continuously for $5 \times 10$ minutes. Several measurements using a Volvo V70 (wheelbase $2.664 \mathrm{~mm}$ ) and a Volkswagen Golv Mk 6 (wheelbase $2.578 \mathrm{~mm}$ ) equipped with a GPSspeedometer were made as reference measurements. 

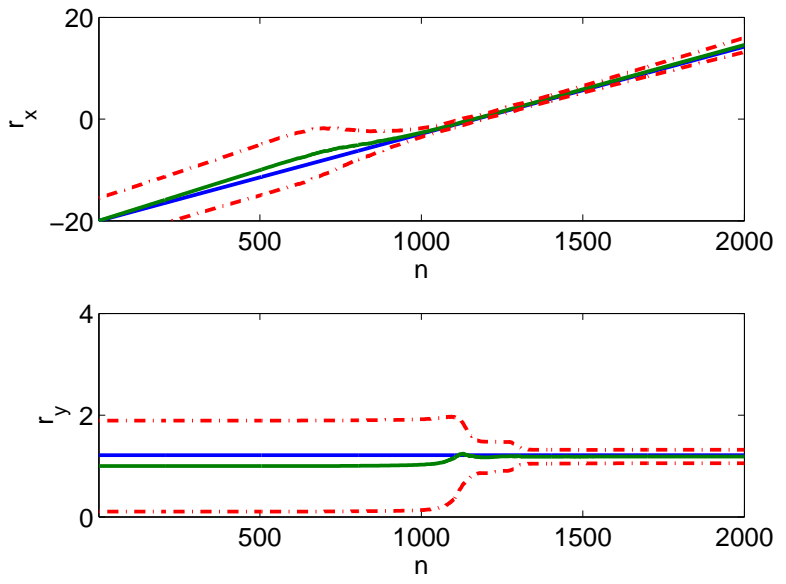

(a)
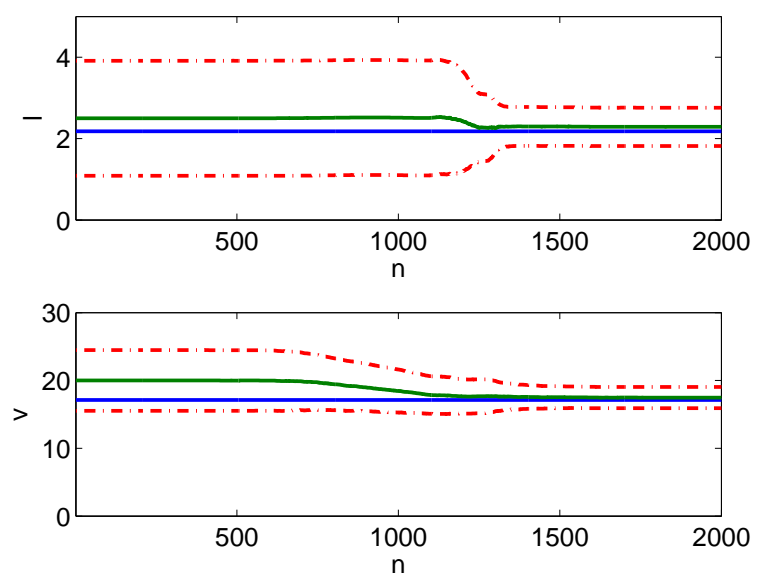

(b)

Fig. 5. Comparison of the true parameter values (blue) and the estimated values (green) together with the $2 \sigma$ confidence interval (red) for (a) $\hat{r}_{x}$ (top) and $\hat{r}_{y}$ (bottom), and (b) $\hat{l}$ (top) and $\hat{v}$ (bottom).

The parameters $\kappa(0.03)$ and $\beta(0.5)$ for one passage of the Volvo V70 at a speed of $69 \mathrm{~km} / \mathrm{h}(19.2 \mathrm{~m} / \mathrm{s})$ were determined and the Kalman filter was applied to the data. The measured vibrations and its envelope together with the estimated envelope are shown in Figure 7.

Figure 8 shows the convergence of the estimated parameters and the corresponding variances. Where known, the true values were also included in the plot (speed and wheelbase). The figures show that the estimates converge rapidly as soon as the signal level rises and becomes stable. The estimated parameters are $\hat{r}_{y}=0.9 \mathrm{~m}, \hat{l}=2.7 \mathrm{~m}$, and $\hat{v}=18.1 \mathrm{~m} / \mathrm{s}$ and are close to the true values.

Similarly, the filter was applied to the other reference measurements. The results are shown in Table II. The filter converges in all the cases except for the last one (VW Golf with $v=26.9 \mathrm{~m} / \mathrm{s}$ ) and the estimation is best for the speed. The filter is somewhat worse for the Golf, mainly due to the more disturbed envelope. Also, further fine-tuning of the initial values based on the measurement data might improve

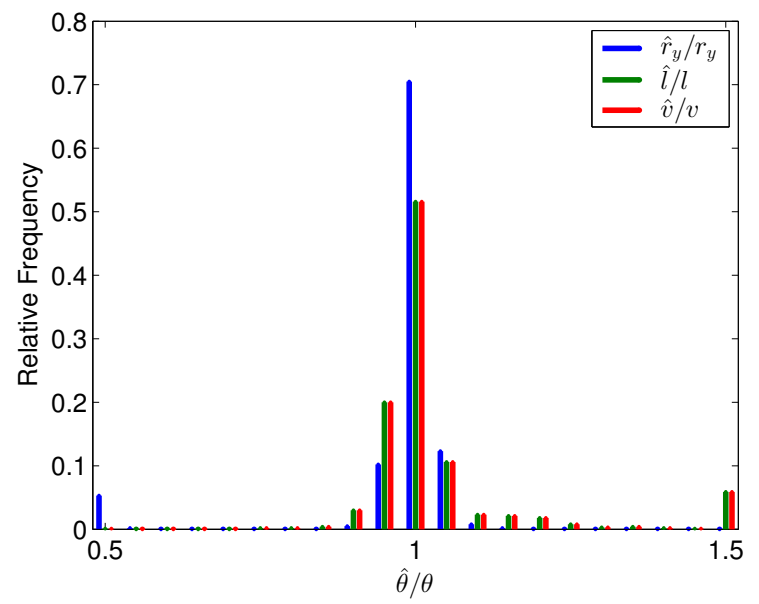

Fig. 6. Relative frequency of the ratios $\hat{r}_{y} / r_{y}, \hat{l} / l$, and $\hat{v} / v$ for 1,000 simulations. The estimates are centered closely around the true values.
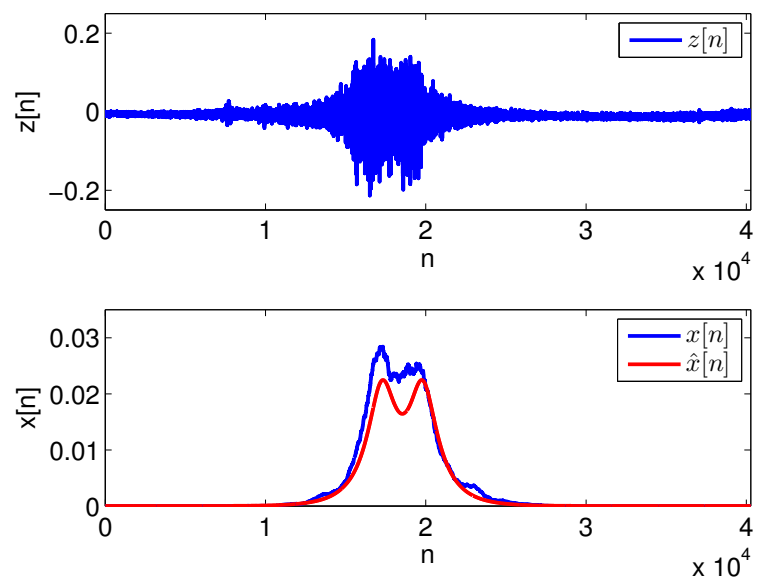

Fig. 7. Measured vibration signal (top), its envelope (bottom, blue), and the estimated signal with the estimated parameters (bottom, red).

the performance.

\section{Discussion}

The preceding results indicate that the proposed method is feasible. The parameters converged towards the real values and at the same time the uncertainty decreased. Clearly, this is not proof that the method works in general - more extensive testing, especially on real data, is needed in order to validate the scheme.

An issue is the choice of the tuning parameters $N_{0}, \alpha$, and $\beta$. The choice of the attenuation parameters is less critical since these can be obtained by calibration and are expected to vary slowly with the ambient conditions only. However, $N_{0}$ may vary from vehicle to vehicle and is thus difficult to find beforehand. More research is needed in order to properly link this to the physical properties.

As with every Kalman filter, especially EKFs, a problem is the choice of the states' priors. If these are chosen badly, the filter is very likely to diverge instead of converge. Possible 
TABLE II
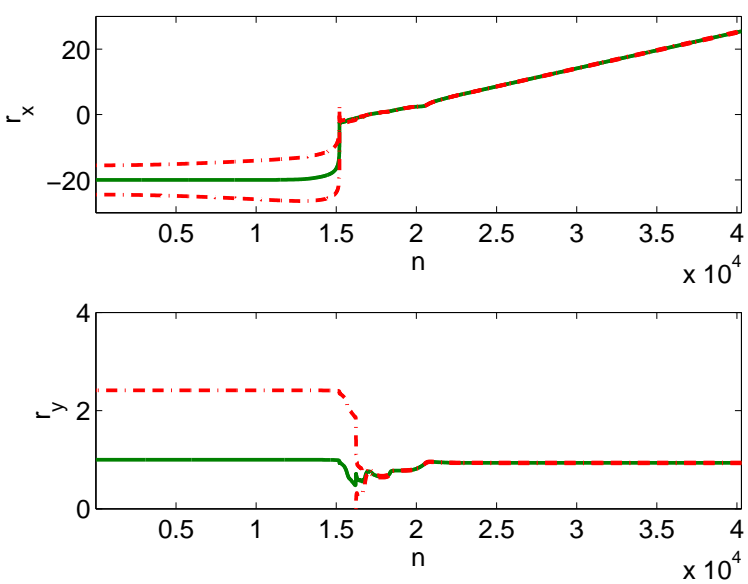

(a)
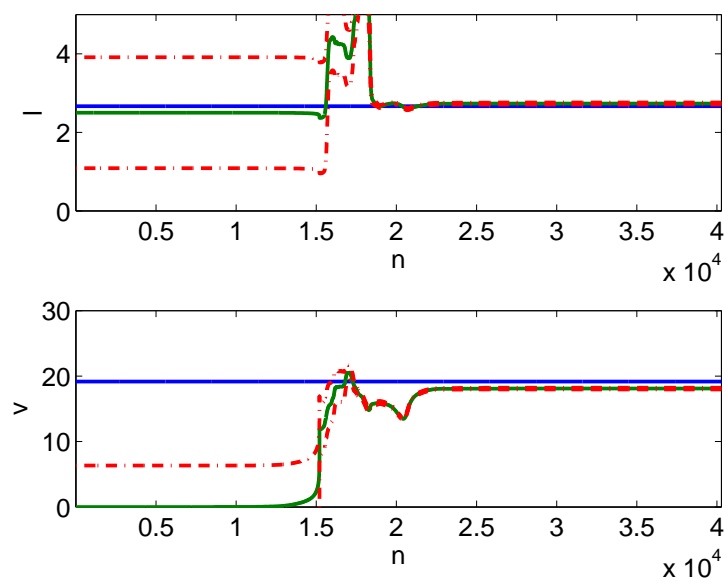

(b)

Fig. 8. Estimation results for a real measurement for (a) $\hat{r}_{x}$ and $\hat{r}_{y}$, (b) $\hat{l}$ and $\hat{v}$ together with the $2 \sigma$ intervals (red).

solutions for this problem might be to either find a generic way of accurately find suitable initial values or to consider a set of range-parametrized EKFs as proposed in [16].

Finally, a worthwhile alternative to consider are particle filters instead of a Kalman filter. As it was mentioned, the distribution of the noise term in (9) is not Gaussian and thus the EKF is suboptimal and a particle filter might produce better results in such a scenario [17].

\section{CONCLUSIONS}

It has been shown in this article that it is in principle possible to use road surface vibrations measured by an accelerometer together with an extended Kalman filter in order to track vehicles moving along a straight road. The theoretical model was applied to measurement data and yielded promising results.

A major issue that has to be resolved in the future is the dependency on the tuning parameters in order to make the scheme fully generic. Possible approaches for solving that challenge have been proposed.
RESULTS FOR THE REFERENCE MEASUREMENTS.

\begin{tabular}{lllll}
\hline Car & $v /(\mathrm{m} / \mathrm{s})$ & $\hat{v} /(\mathrm{m} / \mathrm{s})$ & $\hat{l} / \mathrm{m}$ & $\hat{r}_{y} / \mathrm{m}$ \\
\hline Volvo V70 & 12.8 & 12.4 & 3.0 & 0.9 \\
$(l=2.664 \mathrm{~m})$ & 19.2 & 18.1 & 2.7 & 0.9 \\
& 24.7 & 23.4 & 2.6 & 0.9 \\
VW Golf & 30.8 & 28.9 & 2.0 & 1.6 \\
$(l=2.578 \mathrm{~m})$ & 13.6 & 13.5 & 2.5 & 1.3 \\
& 19.4 & 18.0 & 1.7 & 2.1 \\
& 26.9 & 15.1 & 3.7 & 1.2 \\
\hline
\end{tabular}

\section{REFERENCES}

[1] R. Hostettler, W. Birk, and M. Lundberg Nordenvaad, "Extended Kalman filter for vehicle tracking using road surface vibration measurements," in 51st IEEE Conference on Decision and Control, Maui, December 2012, accepted for publication.

[2] B. Pani, P. Scala, R. Bolla, and F. Davoli, "Filtering and prediction techniques in radar tracking of vehicles for assisted driving," in Image Analysis and Processing, 1999. Proceedings. International Conference on, 1999, pp. 1148-1151.

[3] L. Figueiredo, I. Jesus, J. Machado, J. Ferreira, and J. Martins de Carvalho, "Towards the development of intelligent transportation systems," in Intelligent Transportation Systems, 2001. Proceedings. 2001 IEEE, 2001, pp. 1206-1211.

[4] H. Veeraraghavan, O. Masoud, and N. Papanikolopoulos, "Computer vision algorithms for intersection monitoring," Intelligent Transportation Systems, IEEE Transactions on, vol. 4, no. 2, pp. 78-89, June 2003.

[5] V. Cevher, R. Chellappa, and J. McClellan, "Vehicle speed estimation using acoustic wave patterns," Signal Processing, IEEE Transactions on, vol. 57, no. 1, pp. 30-47, January 2009.

[6] E.-H. Ng, S.-L. Tan, and J. G. Guzman, "Road traffic monitoring using a wireless vehicle sensor network," in Intelligent Signal Processing and Communications Systems, 2008. ISPACS 2008. International Symposium on, February 2009.

[7] E. Sifuentes, O. Casas, and R. Pallas-Areny, "Wireless magnetic sensor node for vehicle detection with optical wake-up," Sensors Journal, IEEE, vol. 11, no. 8, pp. 1669-1676, August 2011.

[8] A. Goel and A. Das, "A brief review on different surface wave methods and their applicability for non-destructive evaluation of pavements," in 2006 Highway Geophysics-NDE Conference, December 2006, pp. 337-350.

[9] A. Burak Goktepe, E. Agar, and A. Hilmi Lav, "Advances in backcalculating the mechanical properties of flexible pavements," Advances in Engineering Software, vol. 37, no. 7, pp. 421-431, 2006.

[10] R. Hostettler, M. Lundberg Nordenvaad, and W. Birk, "A system identification approach to modeling of wave propagation in pavements," in 16th IFAC Symposium on System Identification, Brussels, July 2012.

[11] J. L. Rose, Ultrasonic Waves in Solid Media. Cambridge University Press, 1999.

[12] Z. Zhou, P. Cai, and R. Chen, "Estimating the axle weight of vehicle in motion based on nonlinear curve-fitting," Science, Measurement Technology, IET, vol. 1, no. 4, pp. 185-190, July 2007.

[13] R. Hostettler and W. Birk, "Analysis of the adaptive threshold vehicle detection algorithm applied to traffic vibrations," in 18th IFAC World Congress, Milano, August 2011.

[14] T. J. Rothenberg, "Identification in parametric models," Econometrica, vol. 39, no. 3, pp. 577-591, 1971.

[15] S. M. Kay, Fundamentals of Statistical Signal Processing: Estimation Theory. Prentice Hall, 1993.

[16] N. Peach, "Bearings-only tracking using a set of range-parameterised extended Kalman filters," Control Theory and Applications, IEE Proceedings, vol. 142, no. 1, pp. 73-80, January 1995.

[17] M. S. Arulampalam, S. Maskell, N. Gordon, and T. Clapp, "A tutorial on particle filters for online nonlinear/non-gaussian bayesian tracking," Signal Processing, IEEE Transactions on, vol. 50, no. 2, pp. 174-188, February 2002. 